\title{
TNFAIP3 in circulating and parenchymal myeloid lineage critically controls monocytes, monocytes- derived cells and microglia
}

\section{Francesca Montarolo ( $\sim$ francesca.montarolo@unito.it )}

The University of Manchester Faculty of Biology Medicine and Health https://orcid.org/0000-00023633-8746

\section{Simona Perga}

Neuroscience Institute Cavalieri Ottolenghi

\section{Carlotta Tessarolo}

Neuroscience Institute Cavalieri Ottolenghi

Michela Spadaro

Neuroscience Institute Cavalieri Ottolenghi

\section{Serena Martire}

Neuroscience Institute Cavalieri Ottolenghi

Antonio Bertolotto

Neuroscience Institute Cavalieri Ottolenghi

\section{Research Article}

Keywords: TNFAIP3, inflammation, myeloid cells, microglia and macrophages

Posted Date: December 3rd, 2019

DOI: https://doi.org/10.21203/rs.2.17968/v1

License: (9) This work is licensed under a Creative Commons Attribution 4.0 International License. Read Full License 


\section{Abstract}

Background. The intracellular ubiquitin-ending enzyme TNFAIP3 is one of the most potent inhibitor of the pro-inflammatory NF-kB pathway. Single nucleotide polymorphisms in the TNFAIP3 locus have been associated to autoimmune inflammatory disorders, including Multiple Sclerosis (MS). Previously, we reported a TNFAIP3 down-regulated gene expression level in blood and specifically in CD14+ monocytes obtained from treatment naïve MS patients in comparison to healthy controls (HC). Notably, myeloid cells which include monocytes, exert a key role in the neuro-inflammatory pathogenic process of MS.

Methods. Here, we evaluated the effect of the specific TNFAIP3 deficiency in myeloid cells including monocytes, monocyte-derived cells (M-MDC) and microglia analyzing the lymphoid organs and central nervous system (CNS) of experimental mice. The TNFAIP3 deletion is induced using conditional knockout mice for the myeloid lineage. Flow cytometry and histological procedures were applied to evaluate the immune cell population of spleen, lymph nodes and bone marrow and the microglial cell density in CNS, respectively.

Results. Here, we found that the deletion of TNFAIP3 in myeloid cells induces a reduced body weight, a decrease in the percentage number of M-MDC and of common monocyte and granulocyte precursor cells. We also reported that the deletion of TNFAIP3 in myeloid cells reports an increased microglial cell density in brain.

Conclusions. Collectively, the results suggest that the presence of TNFAIP3 in myeloid cells critically controls the development of M-MDC in lymphoid organ and of microglia in brain.

\section{Background}

Inflammation is an adaptive physiological response to cell injury characterized by the production of proand anti-inflammatory mediators that establish both innate and acquired immune response (1). During physiological conditions, inflammation restore tissue integrity and function. However, in case of prolonged or non-resolving inflammatory events, inflammation can evolve into detrimental process contributing to the pathogenesis of chronic inflammatory disorders, such as autoimmune diseases. Many of the signaling molecules produced by inflammatory events are under the control of the transcription factor nuclear factor kappa-light-chain-enhancer of activated B cells (NF-kB), able to induce inflammatory responses through the activation and the production of pro-inflammatory cytokines, cellular mediators and response genes. In this contest, the intracellular ubiquitin-ending enzyme TNFAIP3 is one of the most potent inhibitor of the NF-kB pathway (2). TNFAIP3 has an ubiquitin ligase C-terminal and a deubiquitinase $\mathrm{N}$-terminal domain (3) and both cooperate to inhibit the NF-kB signaling through the ubiquitination of different NF-kB-signaling molecules.

Single nucleotide polymorphisms (SNPs) in the TNFAIP3 locus have been associated to autoimmune disorders such as systemic lupus erythematous (SLE) (4), rheumatoid arthritis (RA) (4,5), psoriasis (6), type one diabetes (7), coeliac disease (7) and multiple sclerosis (MS) (8). Previously, we reported a 
TNFAIP3 down-regulated gene expression level in the whole blood and in peripheral blood mononuclear cells (PBMCs) obtained from treatment naïve MS patients in comparison to healthy controls (HC) (9-12). Notably, we observed the abnormal expression levels of TNFAIP3 being reverted in patients with MS during pregnancy, which represents a transitory state of immune tolerance associated with reduced disease activity (9). We also demonstrated a negative correlation between the TNFAIP3 gene expression level and the clinical parameters such as the relapse rate and Expanded Disability Status Scale (EDSS) score, indicating that the most aggressive form of MS are characterized by lower level of TNFAIP3 expression (10). Furthermore, we found that the peripheral blood cell population most affected by the reduced expression of TNFAIP3 in MS patients are CD14+ monocytes (13).

Consistently, different studies highlighted an important role of myeloid cells which include monocytes, monocytes-derived cells (M-MDC) such as macrophages and dendritic cells and microglia, in the pathogenic autoimmune process of MS. Indeed, activated macrophages and microglia are hallmarks of active lesions with ongoing demyelination and axonal injury in MS able to induce neuro-inflammation in the brain parenchyma (14).

To date the specific evaluation of the effects of the TNFAIP3 deficiency in monocytes derives from few studies with murine models. Interestingly, mice deficient in TNFAIP3 either completely (15) or in hematopoietic cells (16) developed severe inflammation, hypersensitive to both lipopolysaccharide and TNF-alpha, cachexia and premature death. In contrast, the circulating myeloid-TNFAIP3 deficient mice did not develop similar signs. However, these mice showed spontaneous polyarthritis and high levels of inflammatory cytokines in their serum, consistent with a sustained NF-kB activation (17). Recently, Voet and colleagues investigated the role of TNFAIP3 in microglia using a targeting strategy based on the longevity and capacity of self-renewal of myeloid cells (18). Notably, mice lacking the TNFAIP3 in microglia showed an increased microglial cell number and affects microglial regulation of neuronal synaptic function. The authors also demonstrated that microglia-specific TNFAIP3 deficiency exacerbates the course of the immune-mediated mouse model of MS, represented by the Experimental Autoimmune Encephalomyelitis (EAE). However, the analysis of the effects of TNFAIP3 deficiency in the myeloid cell including M-MDC and microglia is still lacking. Hence, we evaluated the effect of the simultaneously TNFAIP3 deficiency in M-MDC and microglia in lymphoid organs and central nervous system parenchyma.

\section{Methods}

\section{Animals}

All experimental procedures were carried out at the Neuroscience Institute Cavalieri Ottolenghi (NICO, Orbassano), approved by the Ethical Committee of the University of Torino and authorized by the Italian Ministry of Health (authorization number: 59/2016-PR). The experiments have been carried out in accordance with the European Communities Parliament and Council Directives of 24 November 1986 
(86/609/EEC) and 22 September 2010 (2010/63/EU). Mice were housed with a 12 hours light/dark cycle and free access to food/water. Adequate measures were taken to minimize pain and discomfort.

TNFAIP3 $^{\text {flox/flox }}$ mice not commercially available, were obtained from the no-profit RIKEN BioResource Center (Japan). The mice were deposited from Dr. H. Honda (http://www.brc.riken.jp/inf/en/index.shtml, RBRC05494) (19). The transgenic mice carrying the CRE recombinase under the control of the myeloidspecific chemokine $\mathrm{C}-\mathrm{X} 3-\mathrm{C}$ motif receptor $1\left(\mathrm{c} \times 3 \mathrm{cr} 1-\mathrm{CRE}^{+-}\right)$promoter element were purchase from The

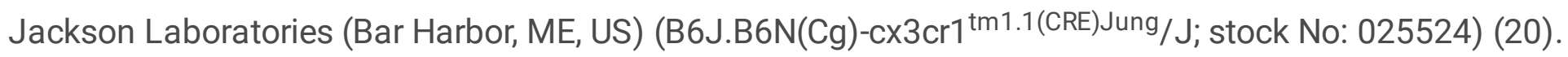
3 months-old female and male TNFAIP3 ${ }^{\text {flox/flox.:. }} \mathrm{cx} 3 \mathrm{cr} 1-\mathrm{CRE}^{+/-}$(TNFAIP3 ${ }^{\mathrm{cx} 3 \mathrm{cr} 1-\mathrm{KO}}$, homozygous knock-out),

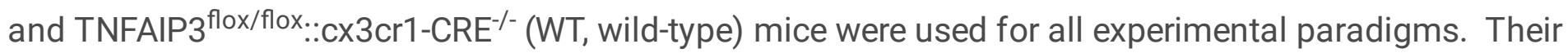
genotype were confirmed by means of polymerase chain reaction (PCR) according to the manufacturer's protocol.

\section{Immunological procedures}

3 months-old TNFAIP3 ${ }^{\mathrm{cx} 3 \mathrm{cr} 1-\mathrm{KO}}(\mathrm{n}=4)$ and WT $(\mathrm{n}=8)$ littermate mice were euthanized by inhalation of isoflurane. Spleen, axillary and inguinal lymph nodes and femoral bone were removed and immersed in fresh phosphate buffered saline (PBS). To collect bone marrow the femurs were cut at both ends and with a $23 \mathrm{G}$ (gauge) needle and a $10 \mathrm{ml}$ syringe filled with ice-cold PBS the bone marrow was flushed out. Tissues (i.e. spleen, axillary and inguinal lymph nodes) were manually homogenized, and rinsed with PBS. The suspended cells obtained from spleen, lymph nodes and bone marrow were centrifuged at 1500 RPM for 10 minutes. The supernatant was discarded and the pellet was suspended in $3 \mathrm{~mL}$ of red blood cells lysis solution (Z3141, Promega, Madison, WI, US) and incubate at $4^{\circ} \mathrm{C}$ for 10 minutes. After adding $10 \mathrm{ml}$ of PBS, the cells were centrifuged at 1500 RPM for 10 minutes. The supernatant was discarded and the cells were cryopreserved at $-80^{\circ} \mathrm{C}$ until use in Roswell Park Memorial Institute (RPMI) 1640 (Invitrogen Life Technologies, Grand Island, NY, US), supplemented with $30 \%$ heat-inactivated fetal bovine serum (FBS, Invitrogen Life Technologies) and 10\% dimethyl sulfoxide (DMSO, Sigma-Aldrich, St Louis, MO, US).

\section{Flow cytometry}

After gentle thawing at $37^{\circ} \mathrm{C}$, the cryopreserved cells obtained from spleen, lymph nodes and bone marrow were immediately added to $5 \mathrm{ml}$ RPMI 1640 (Invitrogen Life Technologies), supplemented with 10\% heat-inactivated FBS (Invitrogen Life Technologies) and centrifuged to remove DMSO. Samples were re-suspended in RPMI 1640 medium supplemented with 10\% heat-inactivated FBS and counted for flow cytometry experiments. Macrophages (i.e CD $11 \mathrm{~b}^{+} \mathrm{F} 4 / 80^{+}$), monocytes (i.e CD11 $\mathrm{b}^{+} \mathrm{Ly}-6 \mathrm{C}^{+} \mathrm{Ly}-6 \mathrm{G}^{+}$), dendritic cells (DCs) (i.e CD $\left.11 \mathrm{c}^{+} \mathrm{CD} 86^{+}\right)$, NK (i.e. $\left.\mathrm{CD} 49 \mathrm{~b}^{+}\right), \mathrm{NK} \mathrm{T}\left(\mathrm{CD}^{+}{ }^{+} \mathrm{CD} 49 \mathrm{~b}^{+}\right)$, T (i.e. $\left.\mathrm{CD} 3^{+}, \mathrm{CD}^{+}{ }^{+} \mathrm{CD} 4^{+}, \mathrm{CD}^{+} \mathrm{CD}^{+}\right)$, and $B$ (ie. $C D 45 R-B 220^{+}$) cells were evaluated in the same spleen and lymph nodes specimen of 3 months-old TNFAIP3 ${ }^{\mathrm{cx} 3 \mathrm{cr} 1-\mathrm{KO}}$ and WT littermates mice. The common myeloid precursors (CMPs, Lin'Sca1

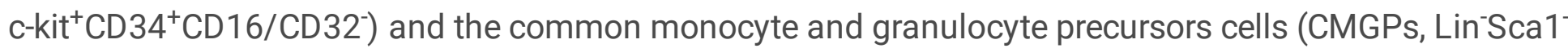
c-kit ${ }^{+} \mathrm{CD} 34^{+} \mathrm{CD} 16 / \mathrm{CD}^{2} 2^{+}$) were evaluated in the same bone marrow specimen of 3 months-old 
TNFAIP3 ${ }^{\mathrm{cx} 3 \mathrm{cr} 1-\mathrm{KO}}$ and WT littermate mice. Non-specific sites of $1 \times 10^{6}$ cells were blocked with rabbit immunoglobulins $\mathrm{G}$ (IgG, Sigma-Aldrich), and cells were then incubated with fluorochrome-conjugated monoclonal $\mathrm{Ab}(\mathrm{mAb})$ and isotype-matched negative controls for 10 min at $4{ }^{\circ} \mathrm{C}$.

The following anti-mouse mAbs were used: CD3-allophycocyanin (APC, 130-109-838), CD45R-B220phycoerythrin (PE, 130-102-292), CD49b- fluorescein isothiocyanate (FITC, 130-102-258), CD11c-APC (130-102-493), F4/80-APC (130-102-379), CD86-PE (130-102-604), c-kit-(CD117)-PE (130-111-693), CD16/CD32-PE (130-102-429) (Miltenyi Biotec, Bergisch Gladbach, Germany), and CD4-phycoerythrin-cy7 (PECy7, BMS25-0041-82), CD8-fluorescein isothiocyanate (FITC, BMS11-0081-82), CD11b-PE (BMS120112-82), Ly6-C-AlexaFluo488 (BMS53-5932-82), Ly6-G-APC (BMS17-5931-82), Lineage cocktail (Lin)PerCP-Cy5.5 (BD Pharmingen, 51-9006964, San Jose, CA, US), Sca1-APC (130-106-425), CD34-FITC (130105-831) (eBioscience, ThermoFisher Scientific, San Diego, CA, US). Living cells identified by propidium iodide (Sigma-Aldrich) exclusion were gated according to their light-scatter properties to exclude cell debris. Samples were analyzed using a CyAn ADP, running Summit 4.3 software (Beckman Coulter, Brea, CA, US).

\section{Histological procedures}

3 months-old TNFAIP3 ${ }^{\mathrm{cx} 3 \mathrm{cr} 1-\mathrm{KO}}(\mathrm{n}=3)$ and WT $(\mathrm{n}=6)$ littermates mice were deeply anesthetized (ketamine $200 \mathrm{mg} / \mathrm{kg}$, xylazine $50 \mathrm{mg} / \mathrm{kg}$ ) and trans-cardially perfused with $4 \%$ paraformaldehyde in $0.12 \mathrm{M}$ phosphate buffer, $\mathrm{pH}$ 7.2-7.4. Brain and spinal cord were removed and immersed in the same fixative at $4^{\circ} \mathrm{C}$ for $24 \mathrm{~h}$ and then cryo-protected in $30 \%$ sucrose in $0.12 \mathrm{M}$ phosphate buffer as reported in (21). Brains and spinal cords were frozen and serially cut by a cryostat in $30 \mu \mathrm{m}$-thick coronal sections collected in PBS. In order to detect microglia, slices were incubated overnight at $4^{\circ} \mathrm{C}$ with the polyclonal anti-rabbit lonized Calcium Binding Adaptor molecule 1 (Iba1, 019-19741, WAKO, Japan) diluted 1:1000 in PBS $1 \mathrm{X}$ with $0.25 \%$ Triton X-100 and $1.5 \%$ normal donkey serum (NDS). The secondary biotinylated antirabbit antibody (715 065 152, Jakson Immuno Research, Philadelphia, PA, US) diluted 1:100 in a solution of PBS containing $0,2 \%$ Triton-X100 and $1,5 \%$ of NDS were used. Immunohistochemical reactions were performed with the avidin-biotin-peroxidase method (Vectastain ABC Elite kit; Vector Laboratories, Burlingame, CA, US) and revealed using 3,3'-diaminobenzidine (3\% in Tris- $\mathrm{HCl}$ ) as chromogen. Images for morphometric analysis were acquired by means of the Nikon Eclipse E600 microscope and analyzed by means of the Cell Counter Tools of ImageJ software (http://rsbweb.nih.gov/ij/index.html). Iba1+ positive $(+)$ cell number (cell number/ $\mathrm{mm}^{2}$ ) in the corpus callosum (Bregma $1.10 \mathrm{~mm}$, Interaural 4.90; Bregma $-0.82 \mathrm{~mm}$, Interaural 2.98) and in the white and grey matter of the cervical level of spinal cord were quantified. At least 3 sections for each animal were evaluated.

\section{Statistical analysis}

Statistical analysis was performed using GraphPad Prism(GraphPad Software, version 5; San Diego, $\mathrm{CA}, \mathrm{US})$. Continuous data were presented as medians and ranges and categorical data were given as 
counts and percentages. Normality of distribution was assessed by the Shapiro-Wilk test. The differences between groups were calculated with Mann-Whitney $U$ test. $p$ values $<0.05$ were considered significant.

\section{Results}

To characterize the phenotype of adult TNFAIP3 ${ }^{\mathrm{cx} 3 \mathrm{cr} 1-\mathrm{KO}}$ mice, their body weight was recorded at 3 months of age. Notably, TNFAIP3 ${ }^{\mathrm{cx} 3 \mathrm{cr} 1-\mathrm{KO}}$ mice $(n=7)$ showed a lower body weight compared to their WT littermates $(n=7)$ (Figure 1, Mann-Whitney test $p=0.014)$. No other macroscopic abnormality were observed in TNFAIP3 ${ }^{\mathrm{cx} 3 \mathrm{cr} 1-\mathrm{KO}}$ mice in comparison to their WT littermates at macroscopic level.

To characterized the immunological phenotype of TNFAIP3 ${ }^{\mathrm{cx} 3 \mathrm{cr} 1-\mathrm{KO}}$ mice due to the absence of TNFAIP3 gene, the cytofluorimetric analysis on sample obtained from spleen (Figure 2) and lymph nodes (Figure 3 ) of adults 3 months old WT $(n=8)$ and TNFAIP3 ${ }^{\mathrm{cx} 3 \mathrm{cr} 1-\mathrm{KO}}(\mathrm{n}=4)$ mice were performed. Notably, the analysis on the spleens highlighted that the percentage number of macrophages (Figure 2A, MannWhitney test $p=0.004$ ), monocytes (Figure $2 B$, Mann-Whitney test $p=0.016$ ), DCs (Figure $2 C$, MannWhitney test $p=0.048$ ) and $B$ cells (Figure $2 F$, Mann-Whitney test $p=0.048$ ) were reduced in TNFAIP3 ${ }^{c \times 3 c r 1-}$ $\mathrm{KO}$ mice compared to their WT littermates. No differences were reported in the other cellular population analyzed, such as NK (Figure 2D), NK T (Figure 2E), CD3+ T lymphocytes (Figure 2G), CD4+ T helper (Figure $2 \mathrm{H}$ ) and $\mathrm{CD} 8+\mathrm{T}$ cytotoxic (Figure 2I) (Mann-Whitney test $\mathrm{p}>0.05$ ) cells. These results indicate that the deletion of TNFAIP3 in myeloid cells leads to an altered immunological phenotype not only in myeloid linage, as expected but also in lymphocytes, such as B cells.

In order to corroborate the results obtained on spleen, the immunological phenotype analysis of lymph nodes obtained from the same micewas performed (Figure 3; WT n=8, TNFAIP3 ${ }^{\mathrm{cx} 3 \mathrm{cr} 1-\mathrm{KO}} \mathrm{n}=4$ ). Notably, TNFAIP3 ${ }^{\mathrm{cx} 3 \mathrm{cr} 1 \mathrm{KO}}$ showed a reduction of the percentage number of macrophages (Figure 3A, MannWhitney test $p=0.006$ ), NK (Figure 3D, Mann-Whitney test $p=0.009$ ) and NK T (Figure3E, Mann-Whitney test $p=0.009$ ) cells in comparison WT littermates. Interestingly, the percentage number of monocytes were not altered in TNFAIP3 ${ }^{\mathrm{cx} 3 \mathrm{cr} 1-\mathrm{KO}}$ mice (Figure 3B, Mann-Whitney test $\left.\mathrm{p}>0.05\right)$. Conversely, DCs (Figure $3 \mathrm{C}$ Mann-Whitney test $p=0.019$ ), B cells (Figure $3 F$, Mann-Whitney test $p=0.019$ ) and $C D 8+T$ cytotoxic (Figure 3 , Mann-Whitney test $p=0.009$ ) were increased in TNFAIP3 ${ }^{\mathrm{c} \times 3 \mathrm{cr} 1-\mathrm{KO}}$ mice in comparison to WT littermates. No differences emerged in the percentage number of CD3+ T (Figure 3G) and CD4+ T helper (Figure 3H) (Mann-Whitney test $\mathrm{p}>0.05$ ) in TNFAIP3 ${ }^{\mathrm{cx} 3 \mathrm{cr} 1-\mathrm{KO}}$ mice in comparison to their WT littermates. Collectively, these results indicate that the lack of TNFAIP3 in myeloid cells impairs also the immunological phenotype at level of lymph nodes.

In order to evaluate whether the immunological alteration occurs during development of immune cells or it is already presents in the precursor cells, the cytofluorimetric analysis was performed also in bone marrow of the same TNFAIP3 ${ }^{\mathrm{cx} 3 \mathrm{cr} 1-\mathrm{KO}}$ and WT mice (Figure 4; WT $\mathrm{n}=8$, TNFAIP3 ${ }^{\mathrm{cx} 3 \mathrm{cr} 1-\mathrm{KO}} \mathrm{n}=4$ ). Specifically, we investigated the consequences of TNFAIP3 ablation in the common myeloid precursors (CMPs, Figure 4A) and in the common monocyte and granulocyte precursor cells (CMGPs, Figure 4B). No differences 
were highlighted in the percentage number of the CMPs between WT and TNFAIP3 ${ }^{\mathrm{c} \times 3 \mathrm{cr} 1-\mathrm{KO}}$ mice (Figure $4 A$, Mann-Whitney test $p>0.05$ ). Conversely, the analysis revealed a decrease of CMGPs in TNFAIP3 ${ }^{\text {cx3cr1- }}$ KO mice compared to their WT littermates (Figure 4B, Mann-Whitney test $p=0.006$ ).

Considering that the TNFAIP3 ${ }^{\mathrm{c} \times 3 \mathrm{cr} 1-\mathrm{KO}}$ mice lead the absence of TNFAIP3 gene also in microglia and that the microglial phenotype has been already studied in the inducible TNFAIP3 ${ }^{\mathrm{cx} 3 \mathrm{cr} 1-\mathrm{KO}}$ mice by Voet and colleagues, we confirmed their results on microglial cell density in the CNS parenchyma. Specifically, the immunohistochemistry analysis was performed in the corpus callosum and in the spinal cord of 3 months-old WT $(n=6)$ and TNFAIP3 ${ }^{\mathrm{cx} 3 \mathrm{cr} 1-\mathrm{KO}}(\mathrm{n}=3)$ littermates. Notably, the evaluated regions represent the most affected areas by demyelination in the human MS and in the EAE murine model. Representative images of coronal section of corpus callosum and spinal cord immunostained with Iba1 antibody were reported in Figure 5. The quantification analysis of the microglial cell density showed that TNFAIP3 ${ }^{\text {cx3cr1- }}$

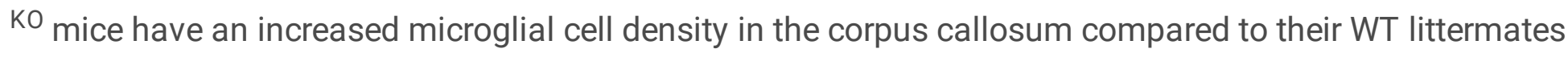
(Figure 6A, Mann-Whitney test $p=0.024$ ). No differences were detected in the microglial cell density of spinal cord between WT and TNFAIP3 ${ }^{\mathrm{cx} 3 \mathrm{cr} 1-\mathrm{KO}}$ mice (Figure 6B-D, Mann-Whitney test $\mathrm{p}>0.05$ ). These data indicate that the lack of TNFAIP3 influence also the myeloid glial compartment.

\section{Discussion}

TNFAIP3 is a central gatekeeper in inflammation and able to inhibit the NF-kB pathway. TNFAIP3 has been genetically associated with different inflammatory autoimmune diseases (4-7), among which MS (8). We previously demonstrated a significant down-regulation of the TNFAIP3 transcript level in peripheral blood obtained from treatment-naïve MS patients in comparison to HC $(9-12,22)$. Further researches revealed that the unbalanced expression of TNFAIP3 in MS was mainly due to the monocyte cell population (13). Interestingly, the analysis of the role of TNFAIP3 in myeloid cells deriving from studies in murine models is still incomplete. In fact, previous work induced the deficiency of TNFAIP3 in M-MDC (17) or in microglia (18), but never in the entire myeloid compartment. Here, we evaluated the effects of the TNFAIP3 lack in myeloid lineage, including M-MDC and microglia in a conditional KO murine model. TNFAIP3 ${ }^{\mathrm{cx} 3 \mathrm{cr} 1-\mathrm{KO}}$ mice were vital and showed a macroscopic normal appearance. Notably, we did not observe in the TNFAIP3 ${ }^{\mathrm{c} \times 3 \mathrm{cr} 1-\mathrm{KO}}$ mice signs of paws inflammation, as reported in the conditional KO model in which the TNFAIP3 gene was deleted only in M-MDC and not in microglia (17). On the other hands, our results are in agreement with the data obtained from Voet and colleagues in which the deficiency of TNFAIP3 in microglia is not related to alteration in gross anatomy of mice. However, we observed a body weight reduction in TNFAIP3 ${ }^{\mathrm{cx} 3 \mathrm{cr} 1-\mathrm{KO}}$ mice compared to their WT littermates. To date, no results related to the body weight in TNFAIP3 KO mice are still reported. In order to detect the specific role of TNFAIP3 in myeloid cells, we performed cytofluorimetric analysis on the spleen of these conditional KO mice. Notably, we revealed, in agreement with previous studies, a strong decreased number of macrophages, monocytes and DCs in TNFAIP3 ${ }^{\mathrm{cx} 3 \mathrm{cr} 1-\mathrm{KO}}$ mice in comparison to WT. Accordingly, in lymph nodes of TNFAIP3 ${ }^{\mathrm{cx} 3 \mathrm{cr} 1-\mathrm{KO}}$ mice we observed a decreased amount in macrophages 
in comparison to WT mice. In addition, we also observed an increased number in DCs, B and CD8+ T cells in lymph nodes of TNFAIP3 ${ }^{\mathrm{cx} 3 \mathrm{cr} 1-\mathrm{KO}}$ mice, in agreement with the studies performed by (17).

These observations suggest that TNFAIP3 is involved in the development of myeloid lineage. In addition, these data also suggest that a de-regulated production of M-MDC due to the lack of TNFAIP3 could be related to general altered immune phenotype involving not only myeloid cells but also lymphocytes, such as $B$ cells and NK.

Thanks to the analysis performed on the bone marrow of the conditional TNFAIP3 ${ }^{\mathrm{c} \times 3 \mathrm{cr} 1-\mathrm{KO}}$ mice, we highlighted for the first time that the immunological alteration did not occur only during the development of immune cells but it was already presents in the precursor cells.

Related to the CNS parenchyma, we observed, in agreement with the Voet's study, an increased microglia cell number in the corpus callosum of TNFAIP3 ${ }^{\mathrm{c} \times 3 \mathrm{cr} 1 \mathrm{KO}}$. However, considering the great analysis performed by Voet and colleagues we decide to not deep insight in this aspect that it has been already fully dissected.

\section{Conclusions}

In conclusion, the obtained results support the suggested role of TNFAIP3 in myeloid cell. In addition to its role in M-MDC, the defects of TNFAIP3 is confirmed to be related to the altered development of other cells population involved in the immune response, such as B and NK. Furthermore, in this study we corroborated previous work (18) reporting an increase in cell density of microglia in CNS parenchyma in TNFAIP3 deficiency condition. Collectively, this work revels a critical role of TNFAIP3 in the control of MMDC and microglia development.

\section{List Of Abbreviations}

- nuclear factor kappa-light-chain-enhancer of activated B cells, NF-kB;

- single nucleotide polymorphisms, SNPs;

- systemic lupus erythematous, SLE;

- rheumatoid arthritis, RA;

- multiple sclerosis, MS;

- peripheral blood mononuclear cells, PBMCs;

- healthy controls, $\mathrm{HC}$;

- expanded disability status scale, EDSS;

- monocytes and monocytes-derived cells, M-MDC;

- experimental autoimmune encephalomyelitis, EAE;

- polymerase chain reaction, PCR; 
- phosphate buffered saline, PBS;

- Roswell Park Memorial Institute, RPMl;

- fetal bovine serum, FBS;

- dimethyl sulfoxide, DMSO;

- dendritic cells, DCs;

- natural killer, NK;

- common myeloid precursors, CMPs;

- common monocyte and granulocyte precursors cells, CMGPs;

- Ionized Calcium Binding Adaptor molecule 1, Iba1;

- normal donkey serum, NDS.

\section{Declarations}

\section{Ethics approval and consent to participate}

No human participants. The statement on ethics approval for animals is included in methods section

Consent for publication

Not applicable

\section{Availability of data and material}

Not applicable

\section{Competing interests}

The authors declare that they have no competing interests

\section{Funding}

The study was supported by the Italian Ministry of Health (Bando Giovani Ricercatori 2010-Grant number GR-2010-2315964) and the Fondazione Italiana Sclerosi Multipla (FISM-Grant number 2010/R/7)

\section{Authors' contributions}

MF, PS and AB designed the study, MF and TS performed the microglia analysis, MF and SM performed the cytofluorimetric analysis, MS analyzed the data, MF wrote the manuscript.

\section{Acknowledgements}


We would like to thank the personnel of the NICO's animal facility

\section{References}

1. Serhan CN, Levy BD, Serhan CN, Levy BD. Resolvins in inflammation: emergence of the pro-resolving superfamily of mediators Find the latest version: Resolvins in inflammation: emergence of the proresolving superfamily of mediators. 2018;128(7):2657-69.

2. Ruland J. Return to homeostasis: downregulation of NF-KB responses. Nat Immunol. 2011;12(8):709-14.

3. Wertz I, Dixit V. A20-a bipartite ubiquitin editing enzyme with immunoregulatory potential. Adv Exp Med Biol. 2014;809:1-12.

4. Shimane K, Kochi Y, Horita T, Ikari K, Amano H, Hirakata M, et al. The Association of a Nonsynonymous Single-Nucleotide Polymorphism in TNFAIP3 With Systemic Lupus Erythematosus and Rheumatoid Arthritis in the Japanese Population. 2010;62(2):574-9.

5. Bowes J, Lawrence R, Eyre S, Panoutsopoulou K, Orozco G, Elliott KS, et al. Rare variation at the TNFAIP3 locus and susceptibility to rheumatoid arthritis. 2010;627-33.

6. Martínez AV, Blanco HG, Flores RC, Muñoz IT, Flores MG, Alanís JS, et al. Candidate gene polymorphisms and risk of psoriasis: A pilot study. 2016;1217-22.

7. Eyre S, Hinks A, Bowes J, Flynn E, Martin P, Wilson AG, et al. Overlapping genetic susceptibility variants between three autoimmune disorders: rheumatoid arthritis, type 1 diabetes and coeliac disease. 2010.

8. Beecham AH, Patsopoulos NA, Xifara DK, Davis MF, Kemppinen A, Cotsapas C, et al. Analysis of immune-related loci identifies 48 new susceptibility variants for multiple sclerosis. Nat Genet. 2013;45(11):1353-60.

9. Gilli F, Lindberg RLP, Valentino P, Marnetto F, Malucchi S, Sala A, et al. Learning from nature:

Pregnancy changes the expression of inflammation-related genes in patients with multiple sclerosis. PLoS One. 2010;5(1).

10. Gilli F, Navone ND, Perga S, Marnetto F, Caldano M, Capobianco M, et al. Loss of Braking Signals During Inflammation. Arch Neurol. 2011;68(7):1-10.

11. Perga S, Martire S, Montarolo F, Navone ND, Calvo A, Fuda G, et al. A20 in Multiple Sclerosis and Parkinson's Disease: Clue to a Common Dysregulation of Anti-Inflammatory Pathways? Neurotox Res. 2017;32(1).

12. Perga S, Martire S, Montarolo F, Giordani I, Spadaro M, Bono G, et al. The footprints of polyautoimmunity: Evidence for common biological factors involved in multiple sclerosis and Hashimoto's thyroiditis. Front Immunol. 2018;9(FEB):1-12.

13. Navone ND, Perga S, Martire S, Berchialla P, Malucchi S, Bertolotto A. Monocytes and CD4+ T cells contribution to the under-expression of NR4A2 and TNFAIP3 genes in patients with multiple sclerosis. J Neuroimmunol. 2014;272(1-2):99-102. 
14. Mishra MK, Yong VW. Myeloid cells - targets of medication in multiple sclerosis. Nat Publ Gr.

15. Lee EG, Boone DL, Chai S, Libby SL, Chien M, Lodolce JP, et al. Failure to regulate TNF-induced NFkappaB and cell death responses in A20-deficient mice. Science. 2000 Sep 29 [cited 2018 May 18];289(5488):2350-4.

16. Nagamachi A, Nakata Y, Ueda T, Yamasaki N, Ebihara Y, Tsuji K, et al. Acquired deficiency of A20 results in rapid apoptosis, systemic inflammation, and abnormal hematopoietic stem cell function. PLoS One. 2014 [cited 2018 May 7];9(1):e87425.

17. Matmati M, Jacques P, Maelfait J, Verheugen E, Kool M, Sze M, et al. A20 (TNFAIP3) deficiency in myeloid cells triggers erosive polyarthritis resembling rheumatoid arthritis. Nat Genet. 2011;43(9):908-12.

18. Voet S, Mc Guire C, Hagemeyer N, Martens A, Schroeder A, Wieghofer P, et al. A20 critically controls microglia activation and inhibits inflammasome-dependent neuroinflammation. Nat Commun. 2018;9(1).

19. Nagamachi A, Nakata Y, Ueda T, Yamasaki N, Ebihara Y, Tsuji K, et al. Acquired deficiency of A20 results in rapid apoptosis, systemic inflammation, and abnormal hematopoietic stem cell function. PLoS One. 2014;9(1):1-10.

20. Yona S, Kim K-W, Haffner R, Jung S. Unraveling Chemokine and Chemokine Receptor Expression Patterns Using Genetically Engineered Mice. In: Methods in molecular biology (Clifton, NJ). 2013. p. $129-44$.

21. Montarolo F, Parolisi R, Hoxha E, Boda E, Tempia F. Early Enriched Environment Exposure Protects Spatial Memory and Accelerates Amyloid Plaque Formation in APPSwe/PS1L166P Mice. PLoS One. 2013;8(7):1-14.

22. Perga S, Montarolo F, Martire S, Berchialla P, Malucchi S, Bertolotto A. Anti-inflammatory genes associated with multiple sclerosis: A gene expression study. J Neuroimmunol. 2015;279(C):75-8.

\section{Figures}




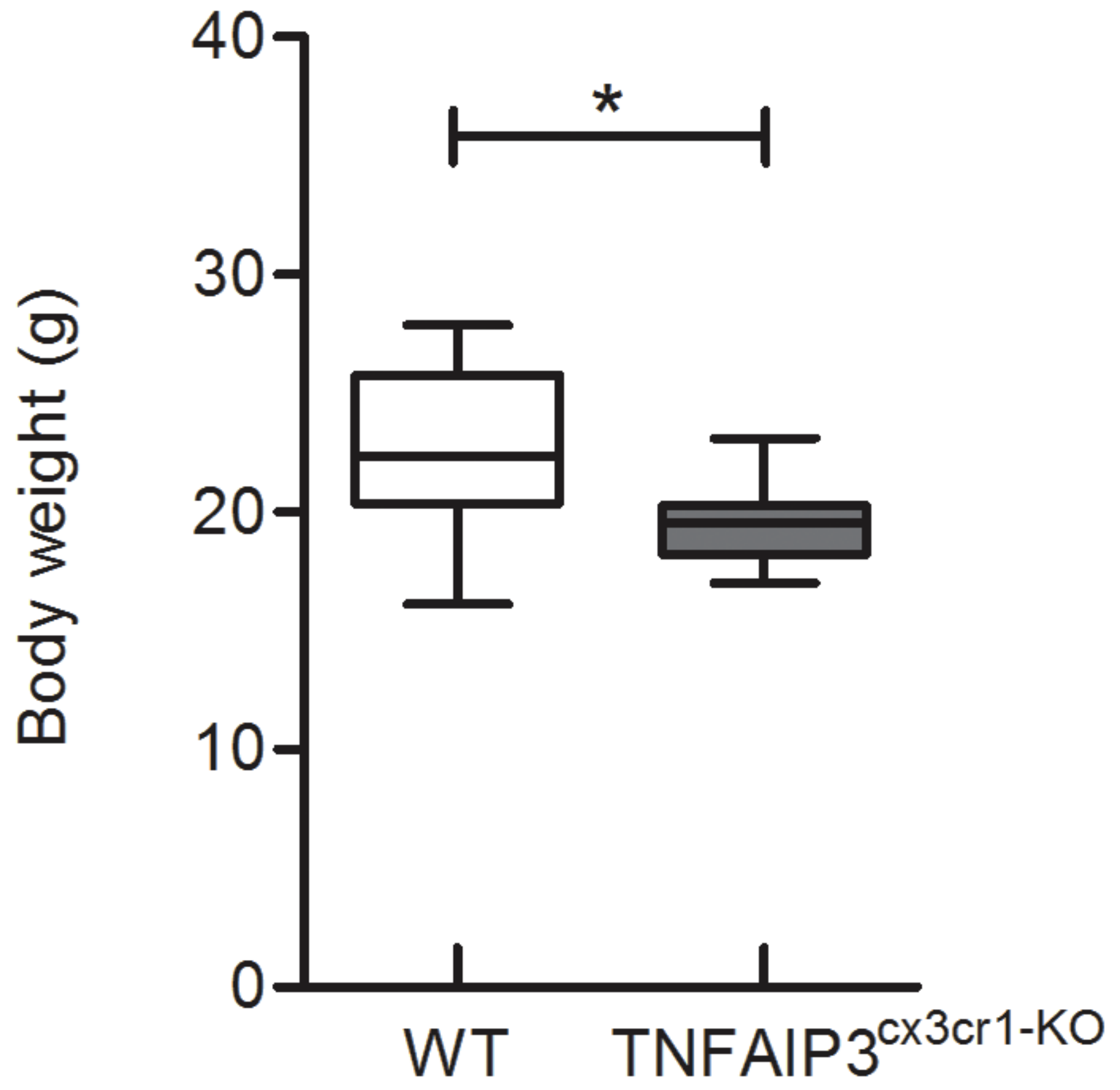

Figure 1

Figure 1. Body weight analysis of 3 months-old WT and TNFAIP3cx3cr1-KO mice. (A) The analysis of body weight reports that at 3 months of age, TNFAIP3cx3cr1-KO mice are smaller compared to their WT littermates ( $n=7$ for each group) (Mann-Whitney test, * $p<0,05)$. 
A

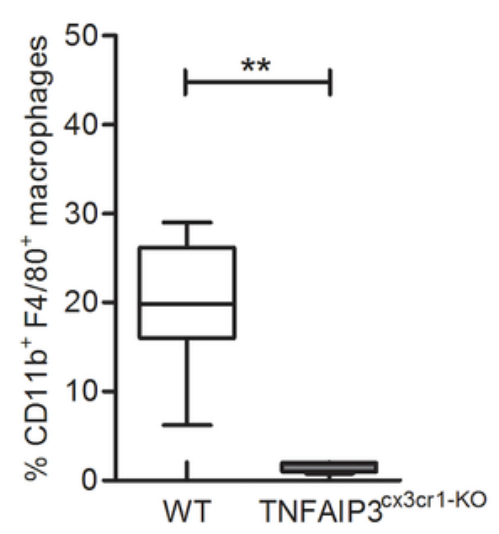

D

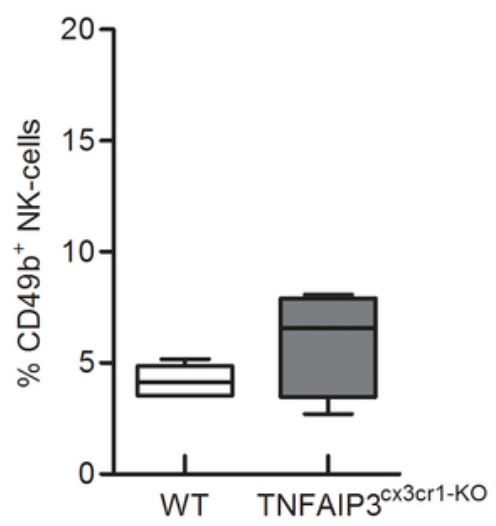

G

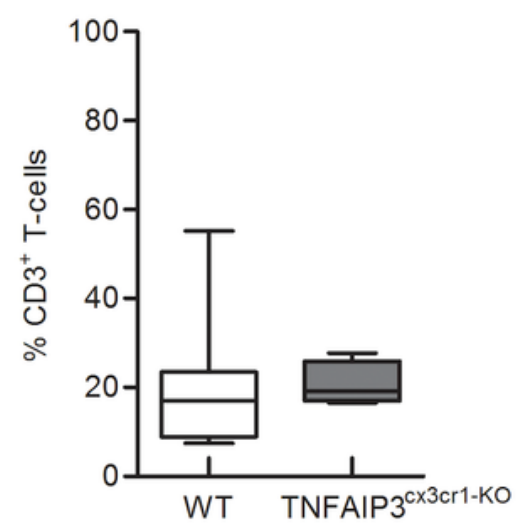

B

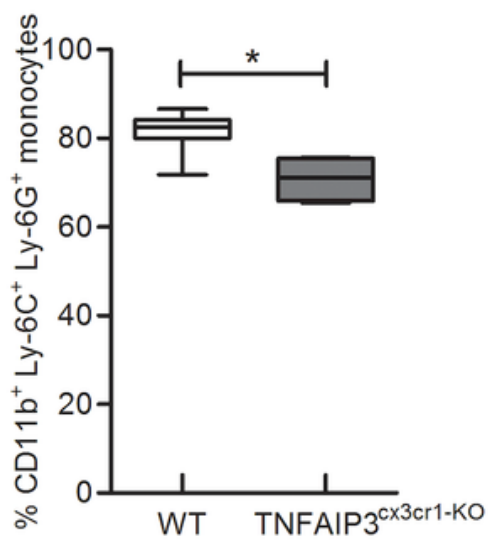

E

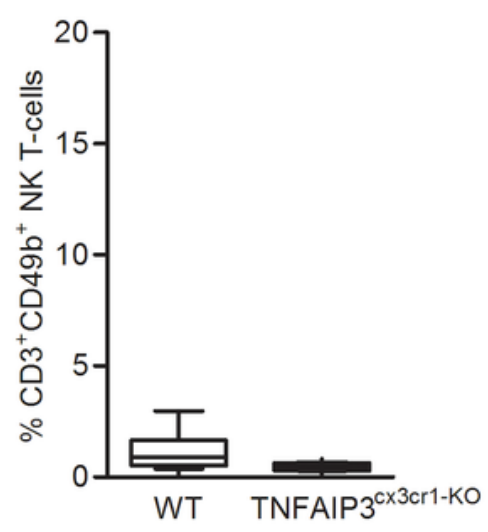

$\mathrm{H}$

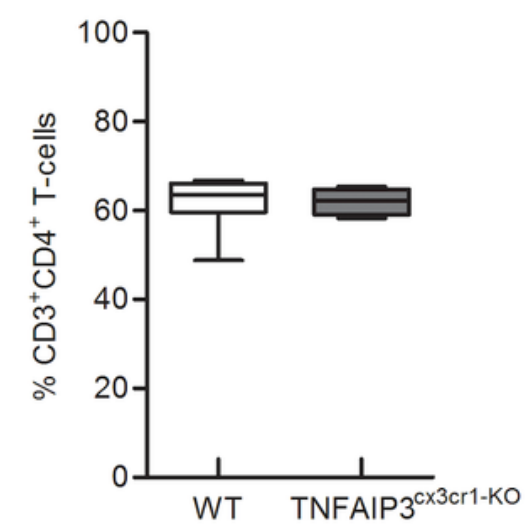

C

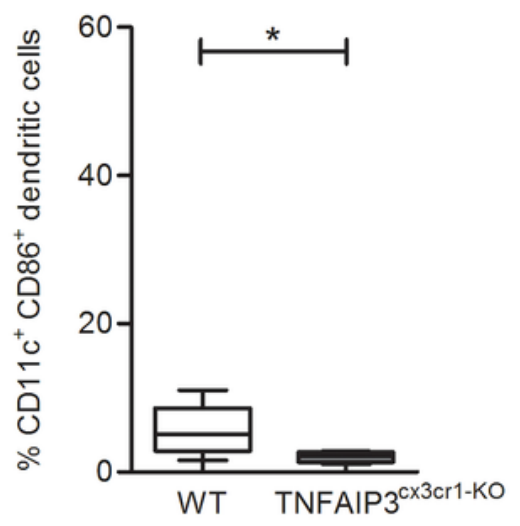

$\mathrm{F}$

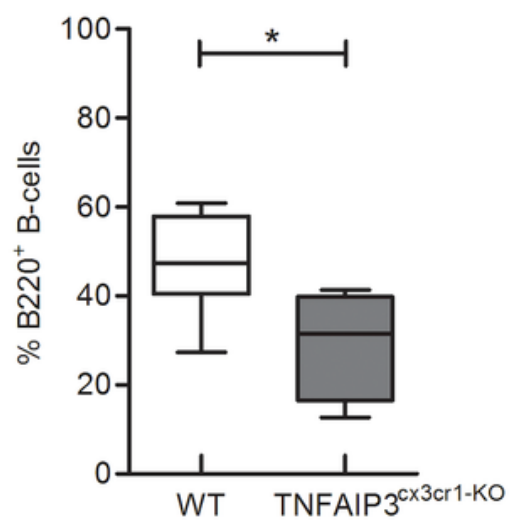

Figure 2

Figure 2. Cytofluorimetric analysis performed on spleen obtained from 3 months-old WT and TNFAIP3cx3cr1-KO mice. The analysis reveals that the percentage number of $\mathrm{CD} 11 \mathrm{~b}+\mathrm{F} 4 / 80+$ macrophages (A), CD11b+Ly-6C+Ly-6G+ monocytes (B), CD11C+CD86+ DCs (C) and B200+B (F) is reduced in TNFAIP3cx3cr1-KO mice compared to their WT littermates. No differences are reported in CD49+NK (D) and CD3+ CD49+NK T cells (E) CD3+ T (G), CD3+CD4+ T helper cells (H) and CD3+CD8+ 
cytotoxic T cells (I) (WT n=8; TNFAIP3cx3cr1-KO n=4) (Mann-Whitney test, ** $p<0,01$; * $p<0,05)$. (DCs, dendritic cells; NK, natural killer).

A

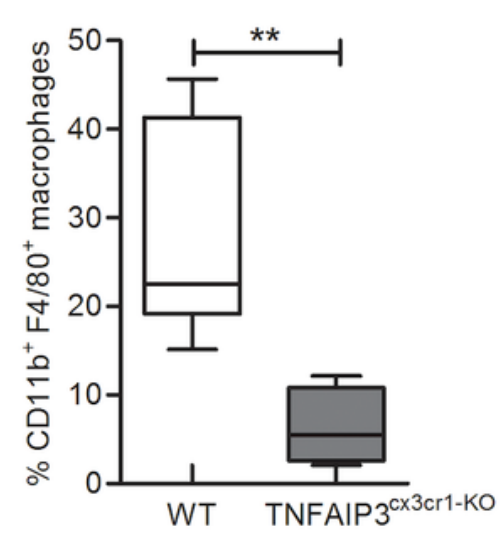

D

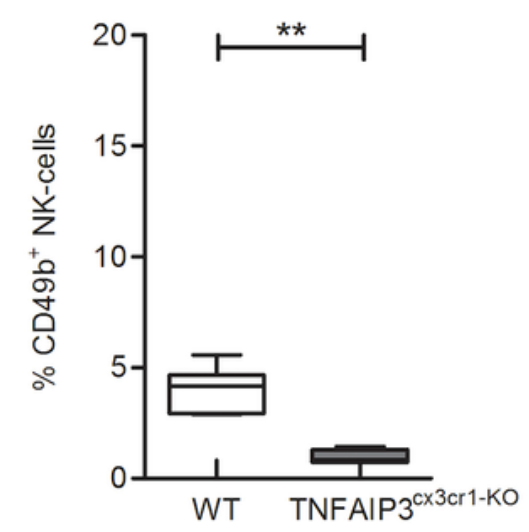

G

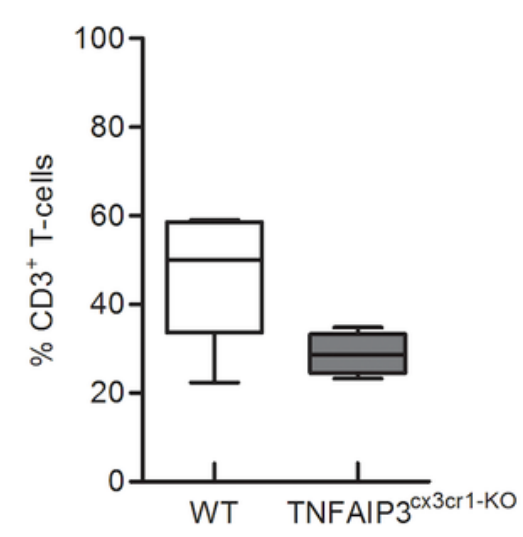

B

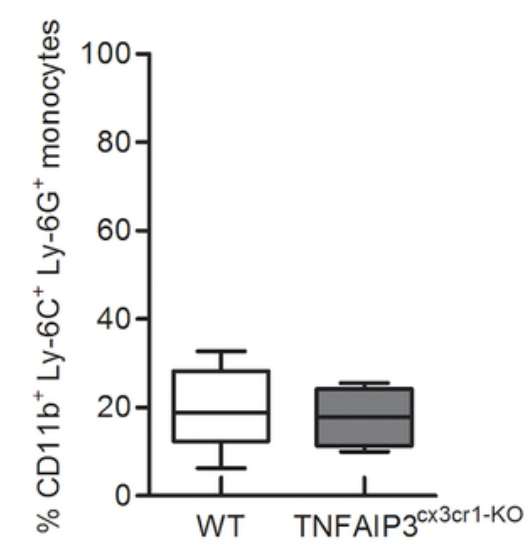

$E$

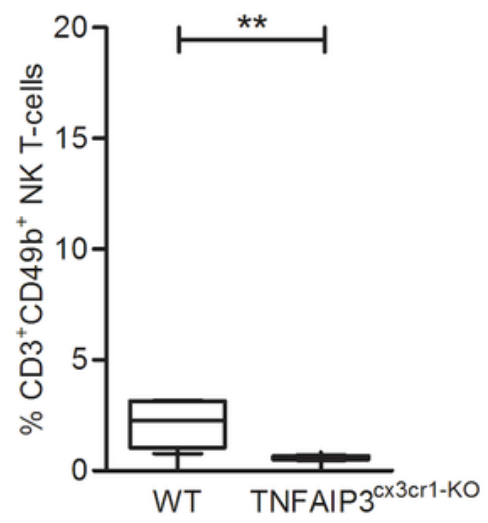

$\mathrm{H}$

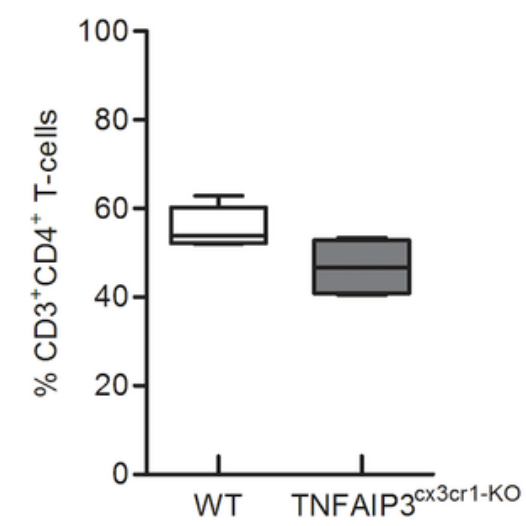

$\mathrm{F}$
C
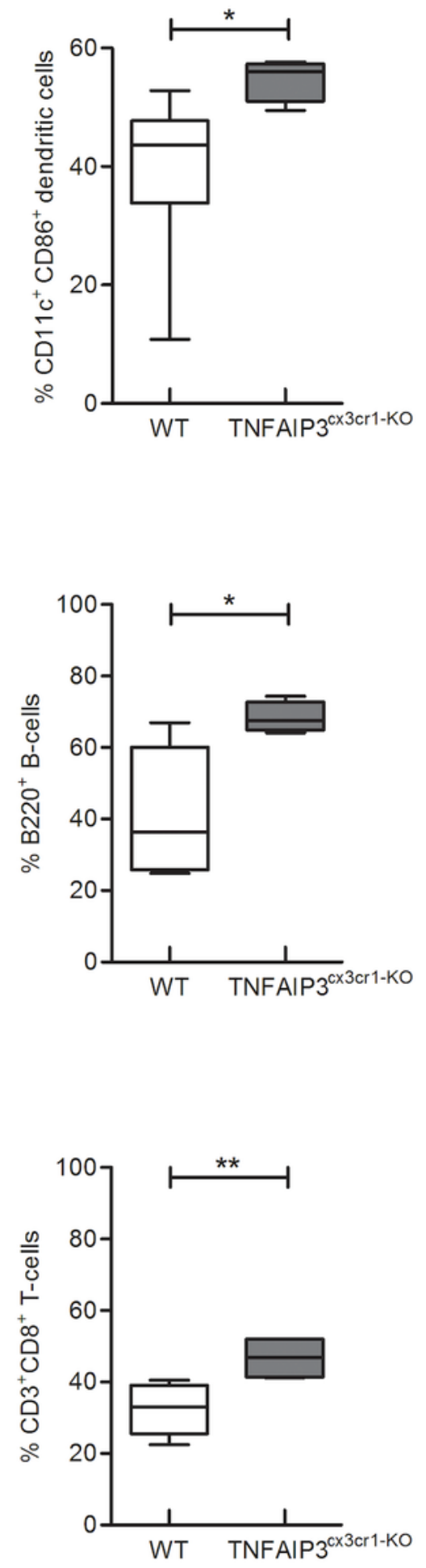

Figure 3

Figure 3. Cytofluorimetric analysis performed on lymph nodes obtained from 3 months-old WT and TNFAIP3cx3cr1-KO mice. The analysis reveals that the percentage number of $\mathrm{CD} 11 \mathrm{~b}+\mathrm{F} 4 / 80+$ macrophages (A), CD49+ NK (D) and CD3+CD49+NK T cells (E) is reduced in TNFAIP3cx3cr1-KO mice 
compared to their WT littermates. No differences are reported in CD11b+Ly-6C+Ly-6G+ monocytes (B), $\mathrm{CD} 3+\mathrm{T}(\mathrm{G})$ and $\mathrm{CD} 3+\mathrm{CD} 4+\mathrm{T}$ helper cells $(\mathrm{H})$. The percentage number of CD11 $+\mathrm{CD} 86+\mathrm{DCs}(\mathrm{C}), \mathrm{B} 200+\mathrm{B}$ $(\mathrm{F})$ and $\mathrm{CD} 3+\mathrm{CD} 8+$ cytotoxic T cells $(\mathrm{I})$ is increased in TNFAIP3cx3cr1-KO mice compared to their WT littermates. (WT $n=8$; TNFAIP3cx3cr1-KO $n=4$ ) (Mann-Whitney test, $* \star p<0,01 ; * p<0,05)$. (DCs, dendritic cells; NK, natural killer).
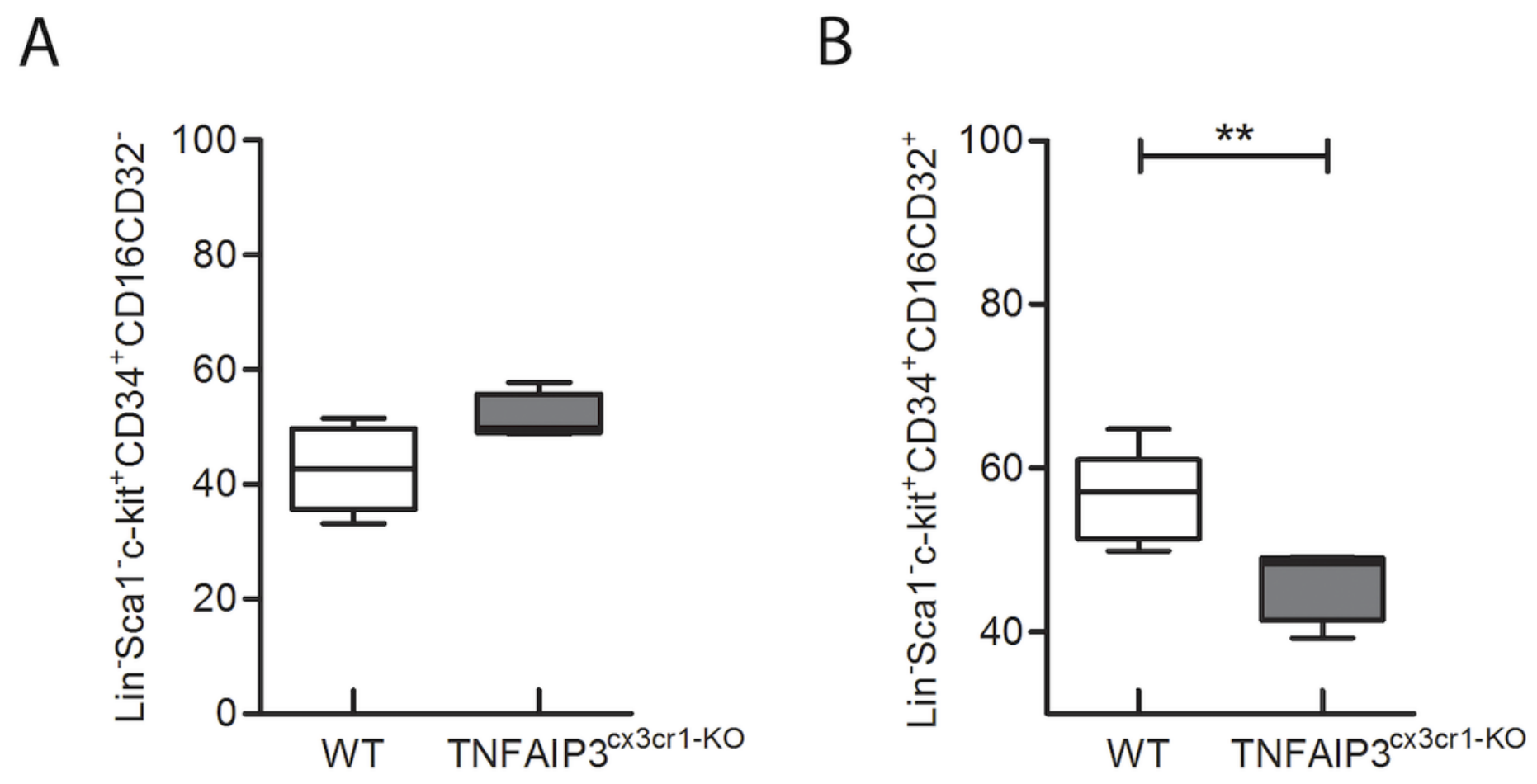

\section{Figure 4}

Figure 4. Cytofluorimetric analysis performed on bone marrow obtained from 3 months-old WT and TNFAIP3cx3cr1-KO mice. No differences are highlighted between WT and TNFAIP3cx3cr1-KO mice in the percentage number of the Lin-Sca1-c-kit+CD34+CD16CD32+CMPs (A). However, the percentage number of the CMGPs (B) is reduced in TNFAIP3cx3cr1-KO mice compared to their WT littermates. (WT $n=4$ and TNFAIP3cx3cr1-KO n=4) (Mann-Whitney test, * $p<0.05)$. (CMPs, common myeloid precursors; CMGPs, common monocyte and granulocyte precursor).

\section{Figure 5}

Figure 5. Iba+ microglial cells in the corpus callosum and in the spinal cord obtained from 3 months-old WT and TNFAIP3cx3cr1-KO mice. (A-D; F-I) Representative images of coronal section of corpus callosum (A-D) and spinal cord (F-I) of WT $(A, C, F, H)$ and TNFAIP3cx3cr1-KO $(B, D, G, I)$ mice immunostained with Iba1 antibody. Calibration bar, $250 \mu \mathrm{m}$ for A-B, F-G; $50 \mu \mathrm{m}$ for C-D, H-I. (E, L) Quantitative analysis of the Iba+ cell density in corpus callosum (E) and spinal cord (L) of WT and TNFAIP3cx3cr1-KO mice disclosed an increased microglial cell density in the corpus callosum of TNFAIP3cx3cr1-KO mice compared to their 
WT littermates. No differences are highlighted in the total spinal cord (L) of TNFAIP3cx3cr1-KO mice compared to their WT littermates. (WT $n=6$ and TNFAIP3cx3cr1-KO $n=3$ ) (Mann-Whitney test, * $p<0.05$ ). (Iba1, lonized calcium binding adaptor molecule 1). 